\title{
Advances in computational modeling through the use of higher-level microstructure characterization
}

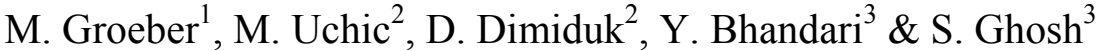 \\ ${ }^{1}$ Department of Materials Engineering, The Ohio State University, USA \\ ${ }^{2}$ Air Force Research Laboratory, MLLMD, Wright-Patterson AFB, USA \\ ${ }^{3}$ Department of Mechanical Engineering, The Ohio State University, USA
}

\begin{abstract}
In this paper, orientation maps of consecutive serial sections are collected in an automated manner by a Focused Ion Beam-Scanning Electron Microscope (FIB-SEM) outfitted with an EBSD system. Micro-Imager, a program developed in this work, uses the 2D EBSD maps to define microstructural features such as grains and grain boundaries. Parameters used to characterize microstructure are also calculated by Micro-Imager for every section. The statistical measurements of each section are compared to assess variability in the microstructure. The 2D sections are reconstructed into a volume by Micro-Imager3D, another program developed in this work. Statistics analogous to those measured in 2D are calculated and compared to the expected distributions predicted by the $2 \mathrm{D}$ measurements coupled with stereology. As a result, quantitative descriptions of microstructure are made and improvements over conventional methods are yielded. Information about individual constituents allows correlations between distributions to be derived. The correlations drawn allow models to account for aspects of microstructure that have classically been overlooked. The resulting 3D grain structure serves as a realistic model microstructure.
\end{abstract}

Keywords: quantitative characterization, equivalent representation, modelling.

\section{Introduction}

The ability to characterize microstructure is an important tool for materials scientists and computational modelers, because it allows one to predict the capability of a material for a given application. For example, it is well known 
that the grain size of a material has a strong effect on mechanical properties; therefore an accurate measure of the grain size distribution is desirable to predict material performance. Classic methods for characterizing microstructure usually involve viewing an image from a sectioned surface, where the area of interest would be mechanically polished [1]. Stereology or other methods can be used to interpolate three-dimensional (3D) statistics from the 2D microstructural images.

However, there are some microstructural parameters that cannot be inferred from 2D sections [2]. In addition, many stereological parameters yield only average values to describe microstructural features. Recognizing the fact that many properties (especially those associated with failure) require extreme values of the microstructure [3], it is evident that characterizing the full distribution of these features may be more appropriate for some predictive models $[4,5]$. This need to more completely characterize microstructure has led to more direct methods, such as serial sectioning, that allow one to obtain true 3D microstructural data [6,7]. For this study, a Focused Ion Beam (FIB) is used to serial-section a specimen and an EBSD system is used to obtain an orientation map of each section. This method shows great potential because the dual beam FIB-SEM microscope can be automated to perform this analysis without user interaction.

Post-processing of the orientation data is performed using a program developed in this study called Micro-Imager. Micro-Imager automatically defines grains and grain boundary segments using the 2D EBSD maps, and calculates statistics of the microstructure based on this information. MicroImager characterizes the microstructure more completely; because it calculates full distributions of parameters in both the 2D sections and the 3D reconstructed volume. This study focuses on the task of developing refined data collection and also improving statistical analysis techniques to increase predictive capabilities. This methodology may be particularly useful for generating a host of statistical microstructural correlations that enable the construction of truly representative material microstructures as input for modeling and simulation programs.

\section{Brief overview of the FIB-EBSD serial sectioning process}

The serial sectioning experiment in the Dual Beam FIB-SEM is comprised of moving the sample repeatedly between two microscope stage positions- the "sectioning" position and the "ion imaging/EBSD analysis" position. Image recognition is critical for precise alignment at the two stage positions, as the microscope stage is only accurate to within few microns when moving between the two stage positions. The image recognition of the DB235 ensures a consistent slice thickness, which is important factor for reconstruction of the serialsectioning data into a 3D volume and is one of the distinct advantages of this automated process. The key advantage of acquiring the orientation information is it allows unsupervised segmentation of grains, which is difficult using only secondary electron or even ion images. A more detailed explanation of the serial sectioning process can be found in [8]. 


\section{3D orientation data reconstruction and grain segmentation and measurement by Micro-Imager}

\subsection{Grain segmentation with Micro-Imager}

Micro-Imager is a newly developed software program that automatically locates and approximates the complex grain boundary positions using a series of line segments and an error-per-unit-length algorithm. This process corrects artifacts that are produced when collecting EBSD data as well as simplifies the structure for subsequent mesh generation for computational modeling.

Grain boundary detection consists of comparing orientations of neighboring data points. The midpoint between two data points is tagged as a boundary point if there is a misorientation of more than 4 degrees. This process is completed for all data points, and then special boundary points are located. Special points are the points that three or more grains share. The initial approximation of the grain boundaries is simply the connection of these special points.

Micro-Imager allows the user to adjust the value of the acceptable error-perunit-length. As the user lowers the acceptable value, the approximated boundaries will have an error-per-unit-length that is intolerable. The specialpoint connection will be changed into two equal-length segments that share an endpoint on a defined boundary point and have the same bounding special points as the initial segment's endpoints. A schematic of the evolving grain boundary approximation is shown in Fig. 1. Low tolerances closer represent the actual data, but do not simplify the structure. Higher tolerances simplify the structure, but it is important not to oversimplify and make erroneous microstructures. A more detailed description of the grain segmentation process is discussed in [8].
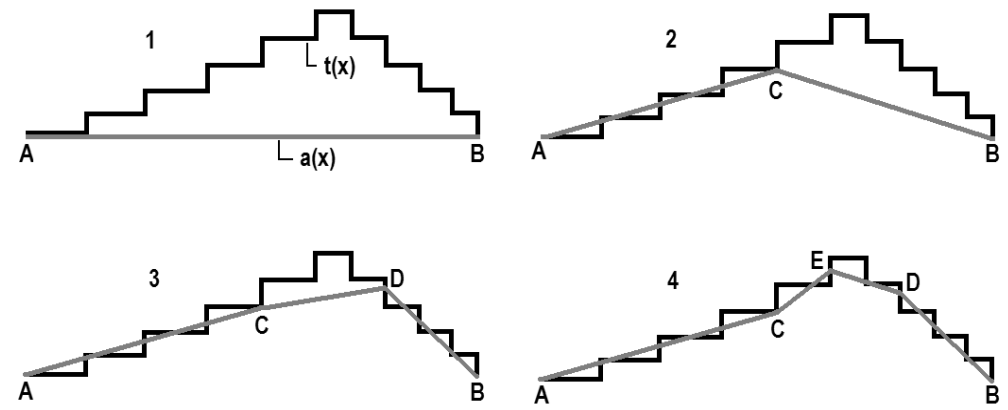

Figure 1: Schematic showing the evolution of the grain boundary approximation in Micro-Imager. Here $t(x)$ represents the true grain boundary shape and the line segment(s) labeled $a(x)$ represent the approximated boundary.

\subsection{Measurement of microstructural parameters by Micro-Imager}

Once Micro-Imager has created a representative microstructure, it has the ability to extract statistical information about the grain morphology. In the initial phase 
of this work, five parameters were selected for measurement. These parameters are characterized in $2 \mathrm{D}$, and corresponding $3 \mathrm{D}$ measurements have also been calculated for some of these parameters. The five parameters measured in $2 \mathrm{D}$ are: misorientation, number of neighboring grains, number of grain boundary edges, grain boundary perimeter, and grain area. Grain volume and number of neighboring grains were also measured in $3 \mathrm{D}$.

\section{Statistical analysis}

\subsection{Micro-Imager analysis versus stereology}

In this work, measurements of two microstructural parameters by Micro-Imager are compared to the same parameters measured by conventional stereology to validate the accuracy of Micro-Imager calculations. The two parameters are grain area and grain boundary surface-area-per-volume.

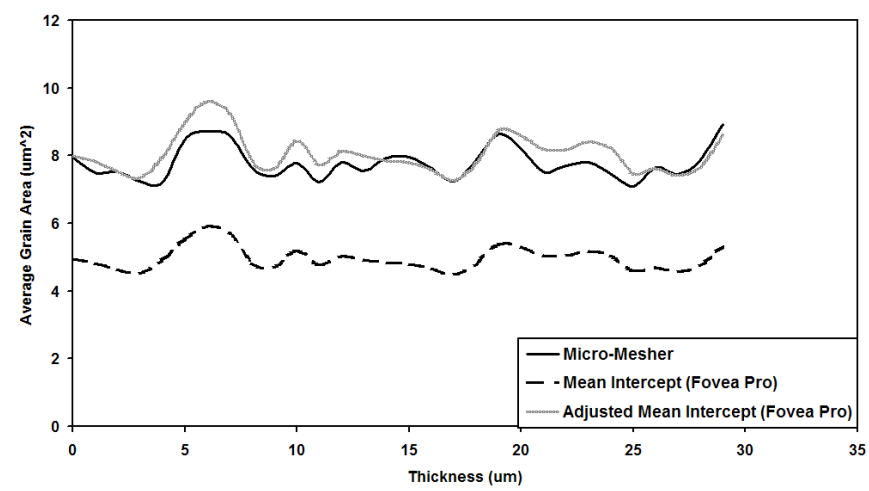

(A)

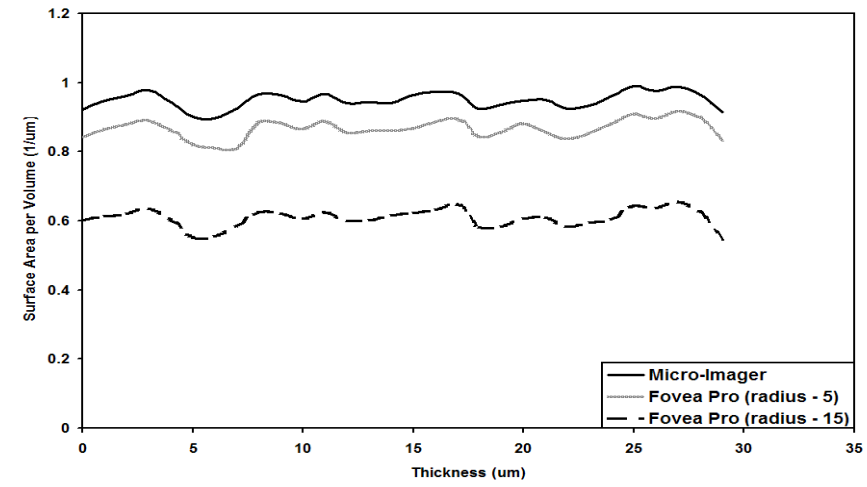

(B)

Figure 2: Comparison of parameter measurements from Micro-Imager and Fovea Pro, where thickness means the sectioning depth. (A) Average grain area and (B) Average grain boundary-surface-areaper-volume. 
The measurement of grain area in Micro-Imager is straightforward. The area of each grain is simply the number of data points originally assigned to that grain multiplied by the area associated with each data point. The calculation also includes the error calculated during grain boundary approximation discussed previously. The stereological measurement of grain area was performed using Adobe Photoshop with the image analysis toolkit Fovea Pro. The grain area was determined using the lineal mean intercept measurement in Fovea Pro.

Figure 2a shows a comparison of average grain area determined by MicroImager and Fovea Pro for section. To calculate the grain area using the lineal mean intercept method, grains were considered to be spherical, i.e. the grain area was assumed to be that of a circle with a diameter equal to the lineal mean intercept. There are two curves for the grain area as calculated by Fovea Pro; the higher curve includes a multiplicative factor that accounts for the error in the lineal intercept method for circular grains in a 2D image [10].

Figure $2 \mathrm{~b}$ shows a comparison of surface area-per-volume measurements. Micro-Imager calculates this value by adding the length of all the grain boundary segments for a grain and dividing by the total area of the grain. A stereological factor is used to translate the value of boundary-length-per-area to boundarysurface-area-per-grain-volume [9]. The calculation using Fovea Pro counts the total number of intersections of a grid of cycloids with the grain boundaries. The number of intersections is divided by the length of the cycloids. This value is multiplied by a stereological factor to convert the intersections-per-length to boundary-surface-area-per-volume [9]. The radius of the cycloids will affect the results, because as the radius approaches zero the intersections approach exactly the grain boundary lines and the cycloids fill the full area, yielding the same value as the length-per-area determined by Micro-Imager. It can be seen in Fig. $2 \mathrm{~b}$ that measurements in Fovea Pro converge to the measurement by MicroImager. This is one advantage of Micro-Imager; that some microstructural parameters can be calculated in a manner that is not sensitive to the measurement method, in contrast to traditional stereological techniques.

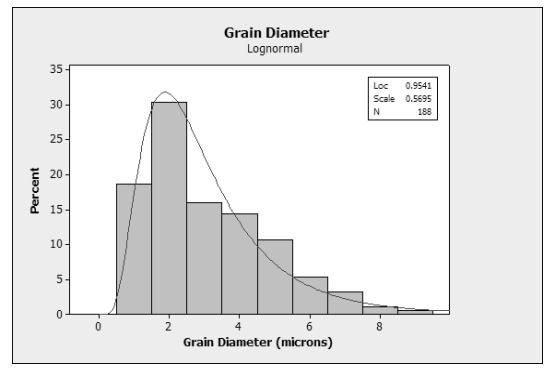

(A)

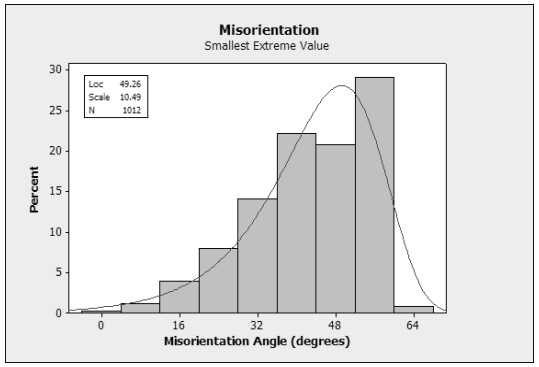

(B)

Figure 3: Probability Density Functions generated by Micro-Imager from a single 2D section. (A) grain diameter (B) misorientation angle. The value of grain diameter is calculated as that of a circle with the same area as the grain. 


\subsection{Probability density functions}

In addition to calculating the mean value of the parameters, the full distributions have been plotted and fitted to determine probability density functions (PDFs), which provide a much more complete characterization. Figure 3 shows the PDFs of grain size and misorientation angle. Probability density functions are useful in creating statistically equivalent microstructures, because they clearly describe the likelihood of a value of a parameter to occur.

\subsection{Parameter variation between slices}

Figure 4 is a 3D surface plot constructed from the series of individual probability density functions. This plot does not substitute for a PDF from a 3D reconstruction, but it can easily display the variability of a parameter through the thickness of the serial sectioning data set. That is, if the PDFs vary markedly, then one $2 \mathrm{D}$ section may not be sufficient to describe a particular microstructural feature.

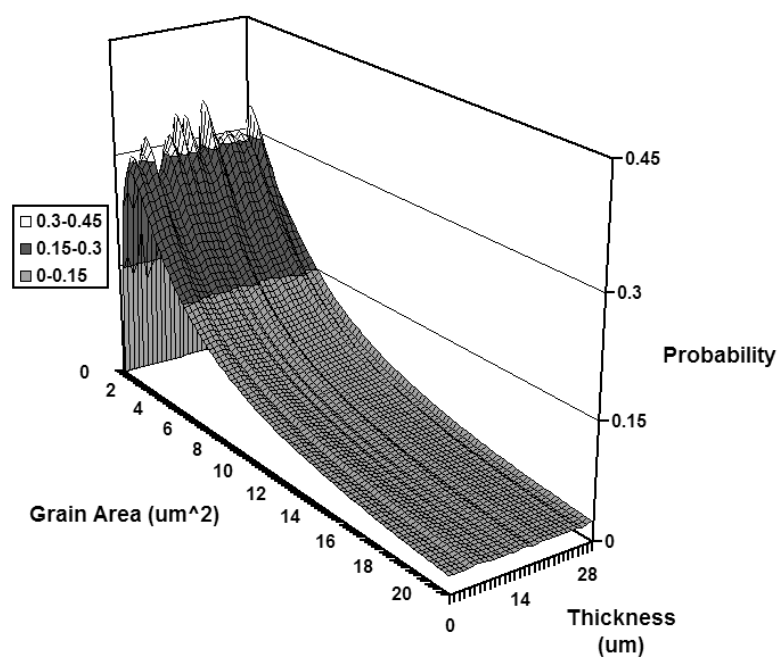

Figure 4: PDFs of grain area shown collectively as a surface plot in 3D to highlight variation from section-to-section.

Two additional applications of this methodology to examine parameter variation are shown in Figs. 5 and 6. In Fig. 5, a histogram of the grain area is plotted, which was constructed by taking the average value of the bin across all the $2 \mathrm{D}$ sections. The error bars denote the maximum and minimum values for each bin across the entire set. The figure illustrates that there can be significant deviations in the distribution of grain size across the 2D images - even for sizes which are at or near the mean. 


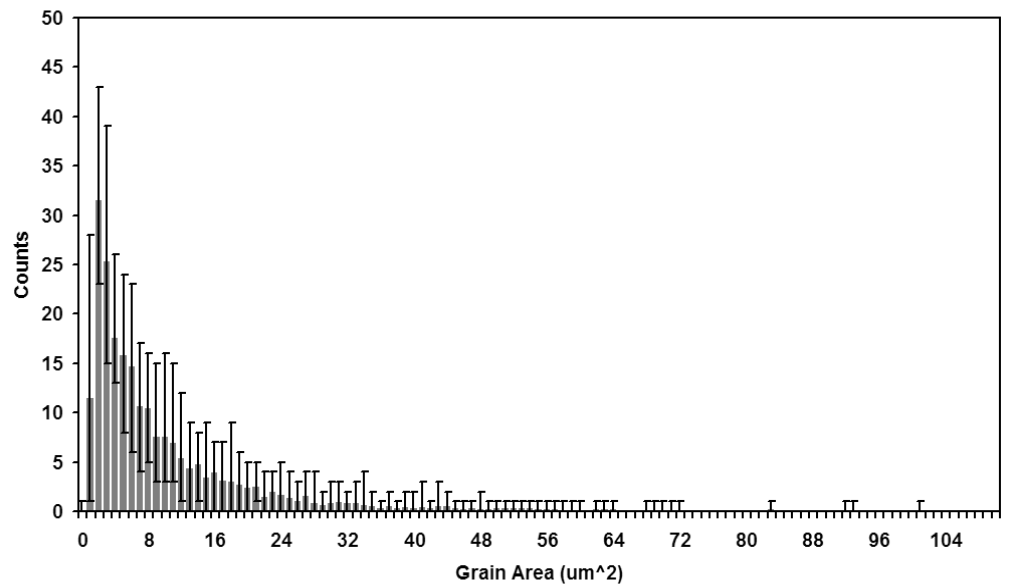

Figure 5: Histogram of grain area that is constructed from the averaged bin values for each size range for all of the sections. The error bars show the maximum and minimum values for each size throughout the entire data set.

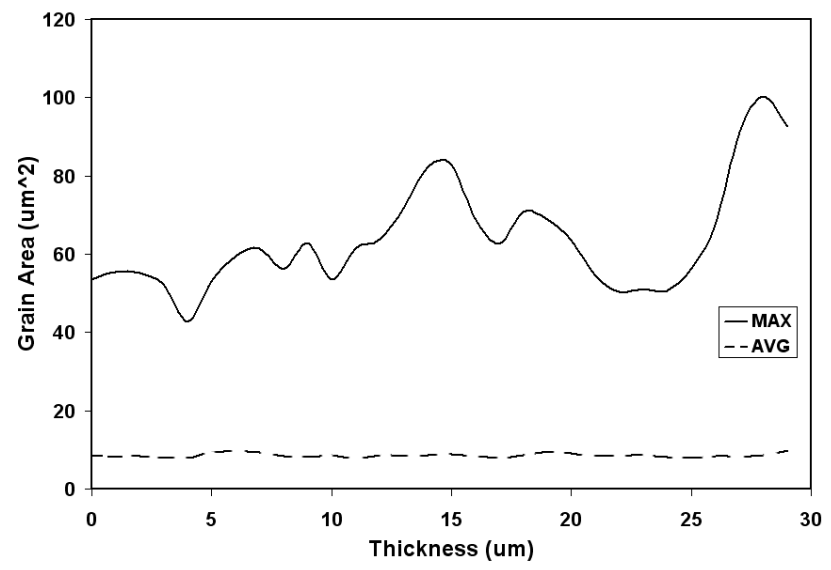

Figure 6: Plot showing the values for average grain area and maximum grain area for each slice.

Figure 6 tracks the average grain area and the maximum grain area on every slice. One can observe that the average grain area appears essentially constant for every slice, but there is noticeable variation in the maximum. The data shown in this section further illustrates the potential need for more detailed microstructural analysis than simply the average of a parameter. Often, the average value for a particular parameter appears constant from slice-to-slice, but can vary markedly in the extreme values. If only the average value of a feature 
is needed for property prediction, then a single section of sufficient size may be adequate. However, if knowledge of the extreme values of a micro-constituent is needed, then the information provided by only one or two sections from a material cannot confidently describe the rest of the microstructure. Furthermore, there are some parameters that can only be calculated from a true $3 \mathrm{D}$ microstructural environment [2].

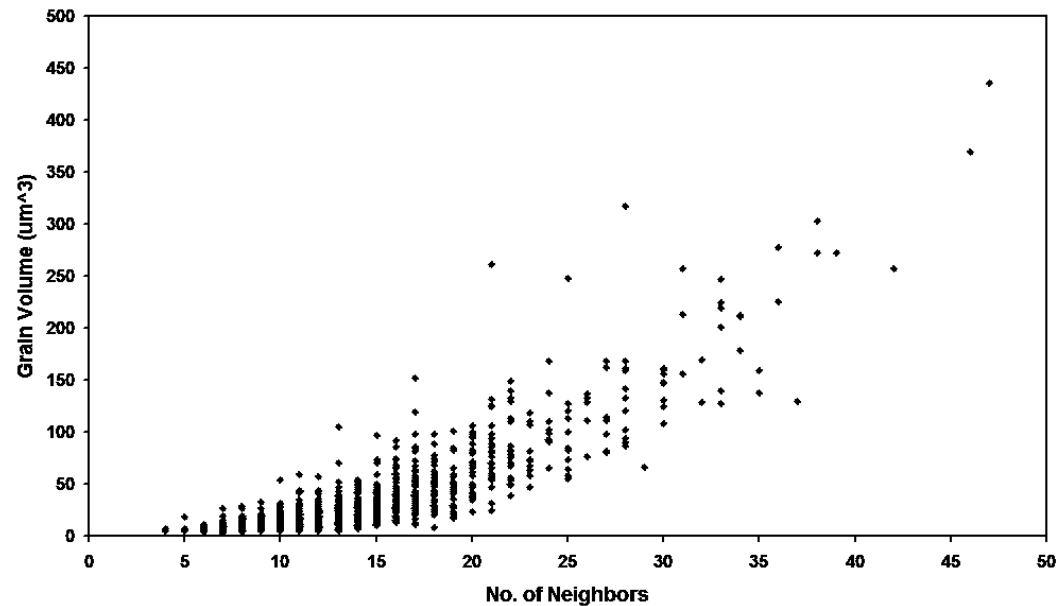

Figure 7: Plot of correlation between grain volume and number of neighboring grains in 3D. Plot shows that small can grains cluster, but large ones tend not to.

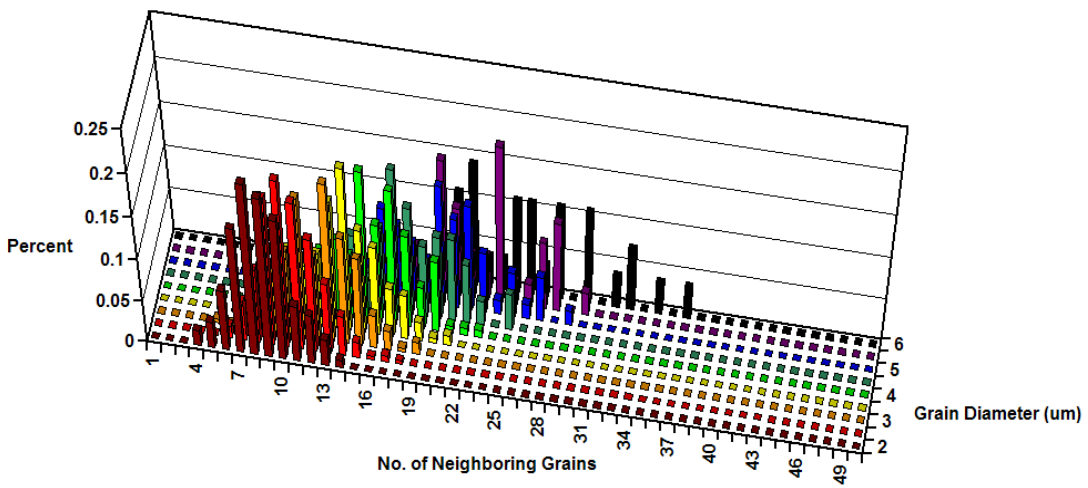

Figure 8: Plot showing the change in the distribution of neighboring grains as grain size increases. 


\subsection{Correlations between microstructural parameters}

In addition to representing distributions of individual microstructural parameters, it may be important for many properties to consider the correlation between multiple parameter distributions. A grouping of similarly oriented grains or a clustering of extremely large or small grains can greatly affect local responses. Figure 7 displays the correlation between grain size and the number of neighbors of the grain. It can be seen in Fig. 7 that small grains can have many neighbors, implying clustering; however, larger grains do not appear to cluster. Accounting for relationships between parameters yields a more accurate description of microstructure. Figure 8 shows distributions of the number of neighbors for various grain sizes. It is clear that as the grain size increases the distributions shift to larger numbers of neighbors as well as broaden. Parameter correlations can yield microstructure models that may produce more realistic results for locally driven properties.

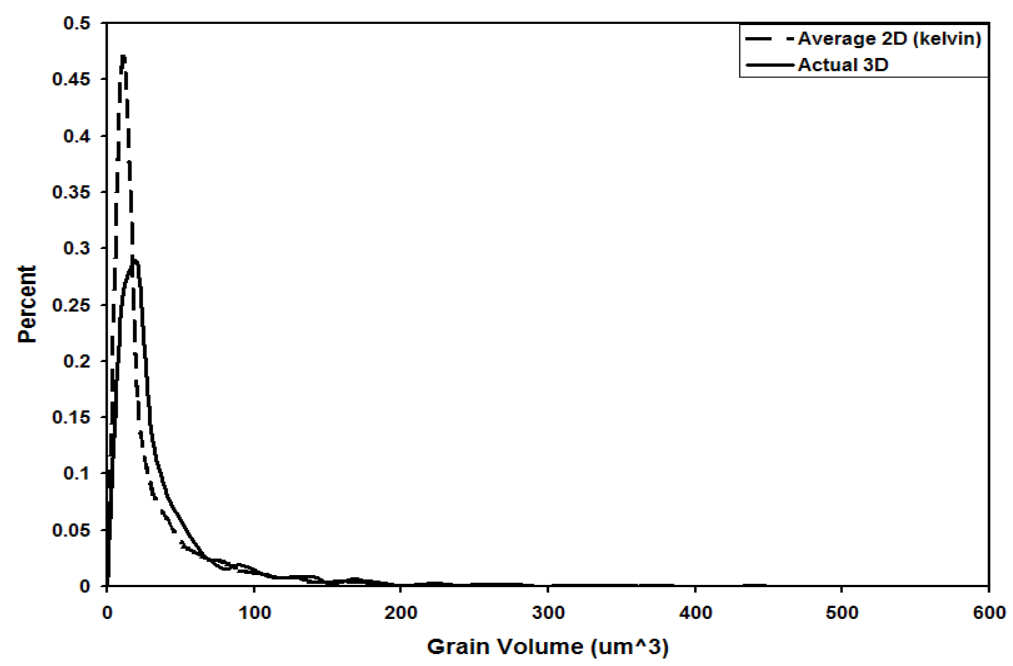

Figure 9: Plot comparing the unbiased distribution of grain volumes from the reconstructed microstructure and the average extrapolated distribution of grain volumes from all the 2D sections.

\subsection{Comparison of 2D and 3D measurements}

The two parameters correlated in Fig. 7, grain volume and number of neighbors, are computed in an unbiased manner from the reconstructed volume. These unbiased measurements can be compared to extrapolated 2D measurements. Comparisons of the two measurements will allow for the evaluation of the extrapolation technique. Figure 9 shows a plot of the unbiased distribution of grain volumes from the reconstructed microstructure along with the average extrapolated distribution of grain volumes from all the 2D sections. Figure 9 
shows that the extrapolated distribution differs noticeably from the actual $3 \mathrm{D}$ distribution. The extrapolation technique used in this comparison, which assumed the grains to be shaped as Kelvin polyhedra, over-predicts the number of very small grains and the size of the largest grains. As a result, the average grain volume for the two distributions is nearly the same, but the extremes of the distributions are quite different.

Parameter measurements on the reconstructed volume provide unbiased descriptions of the grain morphology. Comparisons such as that in Fig. 9 will show where classic stereological relations breakdown. In addition, there are anomalies in the reconstructed volume that maybe deviate significantly from classic assumptions of microstructure. For example, the average number of neighbor grains in the reconstructed microstructure may agree with theoretical calculations, but theoretical calculations greatly under-predict the maximum number of neighbor grains.

\section{Conclusion}

Material characterization is a key in the prediction of material function. The ability to understand the microstructure is the first step in being able to optimize parameters that inevitably lead to superior materials. This study has introduced both experimental and computational procedures that enable new techniques to the forefront of microstructural analysis. This paper presents a snapshot of the representation and analysis methodology as it exists currently. The focus of this work is to continue to improve the representation of microstructures to create realistic computational models for prediction of material capabilities.

\section{References}

[1] Samuels LE. Metallographic Polishing by Mechanical Methods. Metals Park, OH: American Society for Metals; 1982.

[2] DeHoff $\mathrm{R}$ T. Quantitative serial sectioning analysis: preview. $J$. Microscopy 1983; 131: 259-263.

[3] Ghosh S and Moorthy S. Particle Fracture Simulation in Non-Uniform Microstructures of Metal-Matrix Composites. Acta Materialia 1998; 46: 965-982.

[4] Kurzydlowski K J, Ralph B, Bucki J J, and Garbacz A. The grain boundary character distribution effect on the flow stress of polycrystals. Materials Science and Engineering A 1995; 205: 127-132.

[5] Kurzydlowski K J. On the dependence of the flow stress on the grain size distribution in polycrystals. Scripta Metallurgica 1990; 24: 879-884.

[6] Li M, Ghosh S, Richmond O, Weiland H, and Rouns T N. Three dimensional characterization and modeling of particle reinforced MMCs, Part I: Quantitative description of microstructural morphology. Material Science and Engineering A 1999; A265: 153-173.

[7] Li M, Ghosh S, Richmond O, Weiland H, and Rouns T N. Three dimensional characterization and modeling of particle reinforced MMCs, 
Part II: Damage characterization. Material Science and Engineering A 1999; A266: 221-240.

[8] Groeber M, Haley B K, Uchic M D, Dimiduk D M, and Ghosh S. Towards 3D Reconstruction and Characterization of Polycrystalline Microstructures Using a FIB-SEM System. Submitted to Materials Characterization 2005.

[9] Russ J C and Dehoff R T. Practical Stereology. New York: Kluwer Academic/Plenum; 2000.

[10] Thompson A W. Calculation of True Volume Grain Diameter. Metallography 5 1972; 366-369. 